\title{
Hypoxia-Induced Bacterial Translocation in the Puppy
}

\author{
By J.L. Lelli, Jr, R.A. Drongowski, A.G. Coran, and G.D. Abrams \\ Ann Arbor, Michigan
}

\begin{abstract}
- Because hypoxia is one of the most common major stresses to which a neonate is exposed, we postulated that it alone might be the cause of intestinal bacterial translocation, which could be the underlying etiology of neonatal sepsis. An animal model, in which hypoxia is the sole stress, was developed in our laboratory and tested in 18 puppies to determine the effect of hypoxia and reoxygenation on intestinal bacterial translocation. In group $I(n=8)$, following laparotomy and cannulation of the superior mesenteric vein (SMV), the $\mathrm{FlO}_{2}$ was decreased from $21 \%$ to $9 \%$ for 90 minutes followed by reoxygenation at $21 \%$ for 120 minutes. The abdomen was closed and the animals were allowed to recover. After 24 hours the mesenteric lymph nodes (MLNs), spleen, and liver were harvested for bacterial determination and the ileum and jejunum for histological evaluation. Group II $(n=7)$ was treated the same as group I with the $\mathrm{FlO}_{2}$ maintained at $21 \%$. Group III ( $=3$ ) animals were killed, without intervention, for bacterial analysis. In group l, the systemic $\mathbf{P O}_{2}$ decreased by $75 \%$, SMV $\mathbf{P O}_{2}$ decreased by $64 \%$, and oxygen delivery to the small bowel decreased by $80 \%$ in comparison with group II. The mean arterial pressure and cardiac output were not significantly different between group I and group II; however, the mucosal blood flow was decreased by $60 \%(P<.001)$ in group I. Arterial and SMV blood lactic acid levels were unchanged in group $I$ in comparison with group II, suggesting that anerobic metabolism was not initiated in the splanchnic circulation during hypoxia. In group I, products of lipid peroxidation, leukotrienes, thromboxane, and prostaglandins were not significantly changed in comparison with group II. Minimal histological injury was demonstrated in both group I and group II animals; however, the incidence of bacterial translocation to the MLNs in group I $(50 \%, P<.001)$ was significantly higher than in groups II and $I I I(0 \%, P<.001)$. This study demonstrates that severe systemic hypoxia and subsequent reoxygenation does not initiate oxidant-mediated, lipid peroxidation injury to the small bowel mucosa, but does allow bacterial translocation to the MLNs. Thus, hypoxia-induced bacterial translocation could serve as a model for neonatal sepsis without apparent bowel injury.
\end{abstract}

Copyright 1992 by W.B. Saunders Company

INDEX WORDS: Bacterial translocation, hypoxia-induced.

From the Section of Pediatric Surgery and the Pediatric Surgery Research Laboratories, C.S. Mott Children's Hospital; the Section of Pediatric Surgery, University of Michigan Medical School; and St Joseph Mercy Hospital, Ann Arbor, MI.

Presented at the Jens $G$. Rosencranz Resident Competition at the 43rd Annual Meeting of the Surgical Section of the American Academy of Pediatrics, New Orleans, Louisiana, October 26-27, 1991.

Address reprint requests to A.G. Coran, MD, Section of Pediatric Surgery, L2110 Matemal-Child Health Center, University of Michigan Medical Center, Ann Arbor, MI 48109-0245.

Copyright $\odot 1992$ by W.B. Saunders Company

0022-3468/92/2708-0008\$03.00/0
$\mathbf{H}$ YPOXIA AND hypothermia are common physiological stresses to which neonates are exposed. These stresses have been associated with varying degrees of small bowel mucosal injury. ${ }^{1}$ While the precise etiology of the bowel injury remains unclear, major associated factors include direct ischemia, ${ }^{2,3}$ with bacterial invasion of compromised tissue $^{4}$ as well as oral feedings, ${ }^{5,6}$ exchange transfusions, ${ }^{7,8}$ umbilical vessel catheterization, ${ }^{9}$ and indomethacin therapy. ${ }^{10}$

Clinically, neonates with this type of intestinal injury may present in many different ways such as bacteremia alone, septic shock without focal infection, or bowel necrosis and perforation. Histological descriptions of the injured intestine range from focal mucosal ulceration and denudation of villi, to severe transmural necrosis of the bowel wall. ${ }^{1}$

We postulated that these clinical outcomes may be the result of hypoxia-reoxygenation injury to the small bowel mucosa with subsequent translocation of bacteria and resultant sepsis. If this is the pathophysiologic sequence, this injury should be associated with lipid peroxidation secondary to an alteration of iron storage and decompartmentalization of the calcium ion pool. Therefore, an animal model, in which hypoxia is the sole stress, was developed and tested in puppies to determine the effects of hypoxia and reoxygenation on small bowel mucosal physiology and bacterial translocation.

\section{MATERIALS AND METHODS}

\section{Experiment}

Eighteen immature ( 6 week old) mongrel puppies weighing 2 to $3 \mathrm{~kg}$ were divided into three groups. On the experimental day, each puppy received a $2.5 \mathrm{mg} / \mathrm{kg}$ dose of xylazine hydrochloride subcutaneously, followed by $20 \mathrm{mg} / \mathrm{kg}$ of intravenous pentobarbitol. The puppies were intubated and placed on volume ventilation (40 $\mathrm{mL} / \mathrm{kg}$ ) with an $\mathrm{FIO}_{2}$ of $21 \%$. Under local Zylocaine anesthesia, a cutdown on the right and left femoral vessels was performed. The femoral artery was cannulated and monitored by a Waters PRD-15 physiological recorder to measure blood pressure. One femoral vein was canulated with a 5F Pediatric Swan-Ganz Flow-Directed Thermodilution Catheter (Baxter Healthcare Corp, Santa Ana, CA), which was attached to an Oximetric-3 cardiac output computer. The other femoral vein was cannulated and a continuous intravenous infusion of 0.9 normal saline was given at a rate of 30 $\mathrm{mL} / \mathrm{h}$.

Using sterile technique, a midline laparotomy was performed. A small vein along the small bowel mesentery was cannulated with a silastic catheter that was advanced under direct vision into the superior mesenteric vein (SMV). A laser Doppler probe was sutured to the antimesenteric side of the terminal ileum $10 \mathrm{~cm}$ 
proximal to the cecum. The laser probe was attached to a MedPacific LD6000 Laser Doppler capillary perfusion monitor. The SMV catheter was flushed with $2 \mathrm{~mL}$ of 0.9 normal saline containing $100 \mathrm{U} / \mathrm{mL}$ of heparin. The abdomen was then closed except for a $1-\mathrm{cm}$ portion through which the SMV cannula and the laser Doppler probe exited. The animals were then monitored for 30 minutes for stabilization of their blood pressure and arterial blood gases. Arterial and SMV blood gas analysis was performed with a Radiometer ABL3 Blood Gas Analyzer.

In group $\mathrm{I}$ animals $(\mathrm{n}=8)$, the inspired $\mathrm{FIO}_{2}$, measured by a Beckman Model D Oxygen Analyzer, was reduced to $9 \%$ by using a nitrogen washout technique. The $\mathrm{FIO}_{2}$ was maintained continuously for 90 minutes. After 90 minutes, the $\mathrm{FIO}_{2}$ was increased to $21 \%$ for a 2 -hour reoxygenation phase. The entire hypoxic and reoxygenation portion of the study was performed in a neonatal isolette with radiant warming, while core temperatures were monitored with the Swan-Ganz catheter and maintained at $37^{\circ} \mathrm{C}$.

Mean arterial pressure (MAP), cardiac output (CO), arterial and SMV blood gases, and bowel mucosal blood flow were monitored and recorded every 15 minutes during the hypoxia and reoxygenation phases. Blood samples for arterial and SMV lactic acid, arterial lipid peroxidation byproducts, thromboxane $B_{2}$ (TBX), prostaglandin $E_{2}$ (PGE), leukotrienes $B 4$ (LT), tumor necrosis factor (TNF), and interleukin-6 (IL-6) were taken every 30 minutes during hypoxia and dfter 1 and 2 hours of reoxygenation.

After 2 hours of reoxygenation, the SMV catheter and laser Doppler were removed and the abdomen closed using sterile technique. The femoral catheters were removed and the two veins and one femoral artery were ligated. The animals were then allowed to tecover from anesthesia and placed in stccl cages. The cages were warmed by a radiant heat source and the puppies were allowed food and water ad libitum. All recommendations and guidelines for animal use as designated and approved by the University of Michigan Unit for Laboratory Animal Medicine were followed.

After 24 hours, the animals were again anesthetized with xylazine and pentobarbitol; and, under sterile conditions, separate specimens of the spleen, liver and mesenteric lymph nodes (MLNs) were harvested for bacterial identification and quantification. Onc-centimeter sections of the proximal jejunum and distal ileum were harvested and immediately placed in formalin for histological analysis.

Group II $(\mathrm{n}=7)$ animals were treated the same as group I; however, the $\mathrm{FIO}_{2}$ was maintained at $21 \%$ throughout the experiment. Group III $(\mathrm{n}=3)$ animals were killed, without intervention, for bacterial evaluation.

\section{Physiological Measurements}

Small bowel mucosal oxygen delivery was calculated as a percent change from baseline values, which were established during the stabilization phase. The percent change from baseline was calculated using the following formula:

$$
\begin{aligned}
& \text { \% Change in } \mathrm{O}_{2} \text { Delivery } \\
& =\frac{\mathrm{DO}_{2} \text { hypoxia }}{\mathrm{DO}_{2} \text { baseline }} \times 100 \\
& =\frac{\mathrm{BF} \text { bowel hypoxia } \times \mathrm{Hgb} \times \mathrm{SO}_{2} \text { art hypoxia } \times 1.34}{\mathrm{BF} \text { bowel baseline } \times \mathrm{Hgb} \times \mathrm{SO}_{2} \text { art baseline } \times 1.34} \times 100^{*} \\
& =\frac{\mathrm{BF} \text { bowel hypoxia } \times \mathrm{Hgb} \times \mathrm{SO}_{2} \text { art hypoxia }}{\mathrm{BF} \text { bowel baseline } \times \mathrm{SO}_{2} \text { art baseline }} \times 100
\end{aligned}
$$

${ }^{*}$ The unbound free oxygen, which accounts for $0.3 \%$, has been ignored in this equation. where $\mathrm{DO}_{2}=$ oxygen delivery; $\mathrm{BF}=$ mucosal blood flow; $\mathrm{SO}_{2}$ art $=$ arterial oxygen saturation. Mucosal oxygen consumption was calculated as a change from the values determined during the stabilization phase using the following equation:

$$
\begin{aligned}
& \% \text { Change in } \mathrm{O}_{2} \text { consumption } \\
& =\frac{\dot{\mathrm{VO}}_{2} \text { hypoxia }}{\mathrm{VO}_{2} \text { baseline }} \times 100 \\
& =\frac{\mathrm{BF} \text { hypoxia }\left(\mathrm{CO}_{2} \text { arterial }-\mathrm{CO}_{2} \text { smv }\right) \text { hypoxia }}{\mathrm{BF} \text { baseline }\left(\mathrm{CO}_{2} \text { arterial }-\mathrm{CO}_{2} \text { smv }\right) \text { baseline }} \times 100 \\
& \mathrm{BF} \text { hypoxia } \times \mathrm{Hgb} \times 1.34 \\
& =\frac{\times\left(\mathrm{SO}_{2} \text { art }-\mathrm{SO}_{2} \mathrm{smv}\right) \text { hypoxia }}{\mathrm{BF} \text { baseline } \times \mathrm{Hgb} \times 1.34} \times 100 \\
& \times\left(\mathrm{SO}_{2} \text { art }-\mathrm{SO}_{2} \text { smv }\right) \text { baseline } \\
& =\frac{\mathrm{BF} \text { hyoxia } \times\left(\mathrm{SO}_{2} \text { art }-\mathrm{SO}_{2} \mathrm{smv}\right) \text { hypoxia }}{\mathrm{BF} \text { baseline } \times\left(\mathrm{SO}_{2} \text { art }-\mathrm{SO}_{2} \mathrm{smv}\right) \text { baseline }} \times 100
\end{aligned}
$$

where $\dot{\mathrm{VC}}_{2}=$ mucosal oxygen consumption; $\mathrm{BF}=$ mucosal blood flow; $\mathrm{SO}_{2}$ art $=$ arterial $\mathrm{O}_{2}$ saturation; $\mathrm{SO}_{2} \mathrm{smv}=\mathrm{SMV} \mathrm{O_{2 }}$ saturation; $\mathrm{CO}_{2}=$ oxygen content arterial or SMV.

\section{Mediator Assays}

Lactic acid was determined colorimetrically with a procedure previously reported by Barker and Summerson. ${ }^{11}$ Free radical activity was determined by using a fluorescent products assay ${ }^{12}$ while TNF and IL- 6 were determine using bioassay techniques. ${ }^{13-15}$ Arachidonic acid metabolites were also assayed by well-estahlished techniques. ${ }^{16-18}$

\section{Bacterial Translocation}

The MLN, spleen, and liver specimens were wcighed and placed in a sterile glass grinding tube containing sterile brain heart infusion media (BHI), then homogenized. Portions of the MLN, spleen, and liver homogenate and the blood were cultured on blood and laked blood agar plates. The blood agar plates were incubated at $37^{\circ} \mathrm{C}$ for 24 hours in a $5 \% \mathrm{CO}_{2}$ environment. The laked blood agar plates were placed in a sealed container using the BBL Gas Pak PLUS system for anaerobic culturing and incubated for 48 hours at $37^{\circ} \mathrm{C}$. After 24 hours the plates were evaluated for the types and number of each colony. Each colony type was characterized descriptively by Gram stain.

Each aerobic colony was then plated on MacConkey's agar (to inhibit gram-positive organisms) and CNA agar (to inhibit gramnegative organisms). The plates were then incubated aerobically for another 24 hours. The CNA colonies were then subjected to a catalase test. A positive test (bubbles) led to an evaluation by the Staph API system while a negative result (no bubbles) led to an evaluation by the Strep API system. The MacConkey's colonies were evaluated by the oxidase test. A positive test led to an evaluation by the API-NFT (nonfermentors) system. A negative test led to an evaluation by the API-20E enterobacteria evaluation system.

Each aerobic colony was replated on blood agar and laked blood agar and reincubated for 48 hours. This process identified any facultative anaerobic colonies and identified the pure aerobic colonies. These colonies were then evaluated by the API-Anerobe system along with the initial colonies that grew under anaerobic conditions.

All results are reported in CFU/g (colony forming units per gram of tissue) for each type of colony and each type of tissue (MLN, spleen, liver, or blood). 


\section{Morphological Analysis}

One-centimeter portions of the jejunum and terminal ileum were obtained and immediately immersed in $10 \%$ buffered formalin. The tissues were embedded in parrafin for sectioning and evaluation following hematoxylin and eosin staining. The specimens were then evaluated by a pathologist who was blind to which groups the specimens came from. The specific criteria evaluated were the degree of subepithelial edema, degree of lamina propria edema, leukocyte infiltration, denudation of the villi, and the degree of vascular congestion.

\section{Statistics}

Statistical analysis was performed on the Michigan Interactive Data Analysis System (MIDAS) using univariate and repeated measures analysis of variance, with $P$ values less than 0.05 considered significant.

\section{RESULTS}

\section{Physiological Response}

Group I systemic $\mathrm{PO}_{2}$ levels decreased $75 \%$ (118 $\mathrm{mm} \mathrm{Hg}$ to $30 \mathrm{~mm} \mathrm{Hg}$ ) while $\mathrm{SMV} \mathrm{PO}_{2}$ decreased $64 \%$ (55 $\mathrm{mm} \mathrm{Hg}$ to $20 \mathrm{~mm} \mathrm{Hg}$ ) during the hypoxic phase. Arterial and SMV oxygen partial pressures returned to baseline values immediately after the reoxygenation phase was begun.

There was a marked reduction of $60 \%$ in mucosal blood flow in group I (compared with group II) during hypoxia $(P<.001)$. The reduction occurred gradually, reaching a nadir after 60 minutes of hypoxia. Recovery of mucosal blood flow after reoxygenation was slow, requiring 2 hours of reoxygenation to return to baseline levels (Fig 1 ).

The combination of decreased systemic $\mathrm{PO}_{2}$ and a decreasing mucosal blood flow resulted in a marked

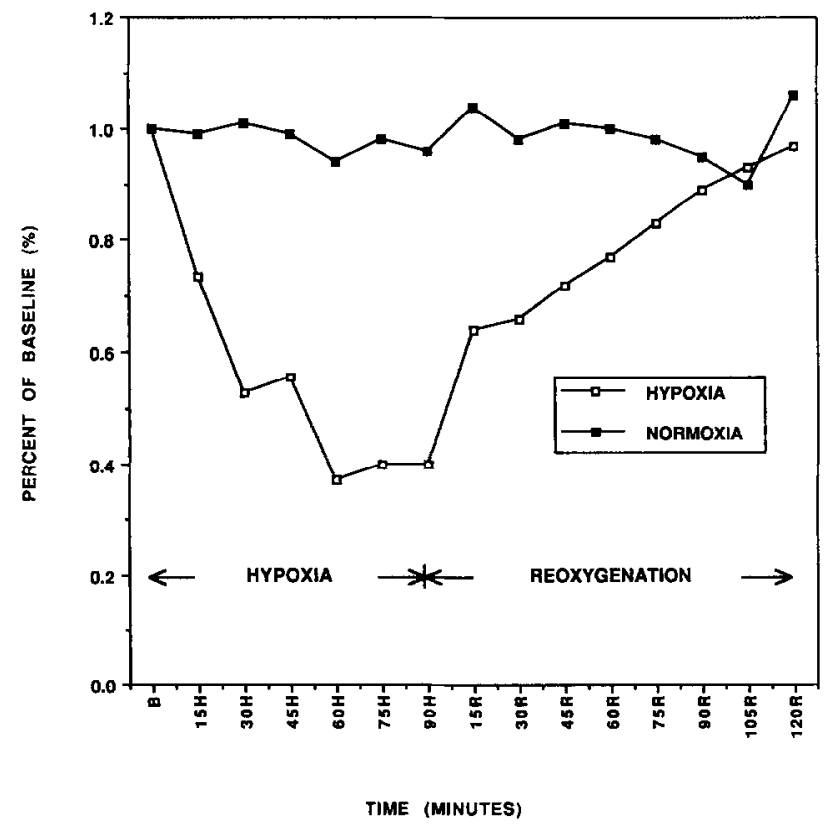

Fig 1. Mucosal blood flow.

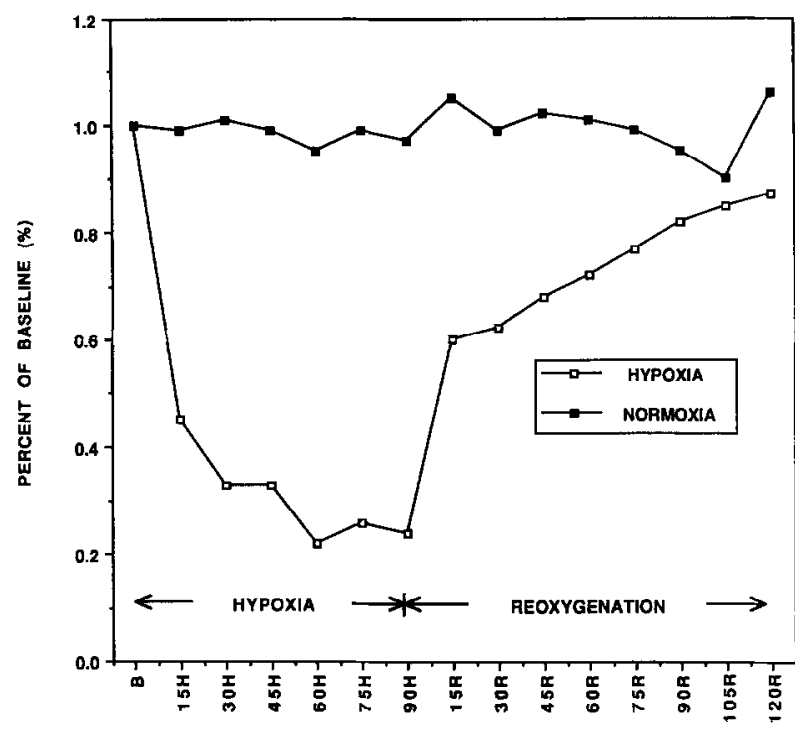

TIME (MINUTES)

Fig 2. Mucosal oxygen delivery.

reduction in oxygen delivery of $80 \%$ (Fig 2). Even though reoxygenation occurred after 90 minutes of hypoxia, oxygen delivery did not rcturn to bascline until 2 hours after reoxygenation was initiated. This phenomenon was primarily due to the slow recovery in mucosal blood flow.

A plot of the average mucosal oxygen consumption $\left(\mathrm{V}_{2}\right)$ versus the average mucosal oxygen delivery $\left(\mathrm{DO}_{2}\right.$ ) of group I animals (Fig 3A) demonstrates that a decrease of greater than $70 \%$ in mucosal oxygen delivery can occur before $\mathrm{O}_{2}$ consumption falls and anaerobic metabolism is initiated. Oxygen delivery did not decrease by $70 \%$ until after 60 minutes of

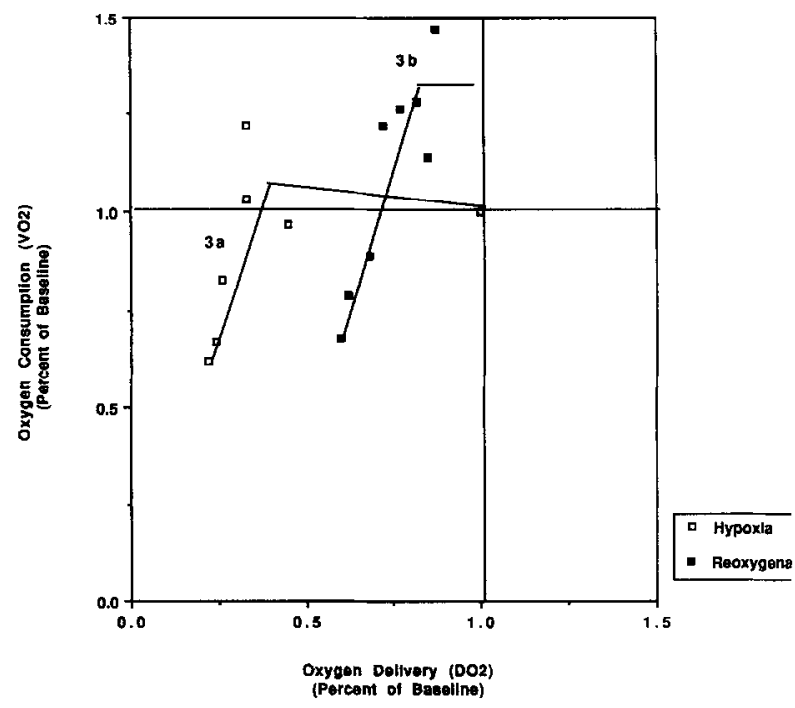

Fig 3. Oxygen consumption versus delivery. $\square$, Hypoxia; $\square$, reoxygenation. 
hypoxia (Fig 2). The anaerobic state was then maintained for only 30 minutes.

When reoxygenation started, a second $\mathrm{VO}_{2}-\mathrm{DO}_{2}$ profile occurred (Fig 3B). Even though normal $\mathrm{PO}_{2}$ levels were being supplied to the mucosa, baseline $\mathrm{O}_{2}$ delivery could not be achieved because of the slow recovery in mucosal blood flow. As mucosal blood flow recovered, a state of overconsumption occurred, characterized by an increase in oxygen extraction.

The CO and MAP were not significantly affected by hypoxia.

\section{Anaerobic Metabolism}

Anaerobic metabolism is necessary for the production of substrates for formation of free radicals following reoxygenation. Arterial and SMV blood lactic acid levels were measured. An increase in lactic acid levels was seen but this increase was not statistically significant $(P>.05$, group I $v$ group II; Figs 4 and 5). Therefore, significant anaerobic metabolism was not initiated in the mucosal circulation, even after severe systemic hypoxia.

\section{Free Radical Activity}

Serum levels of fluorescent products (lipid peroxidation or free radical activity) showed a slight increase in group I compared with group II; however, this was not statistically significant. Therefore, the hypoxic stress and subsequent reoxygenation in this model was not capable of initiating free radical

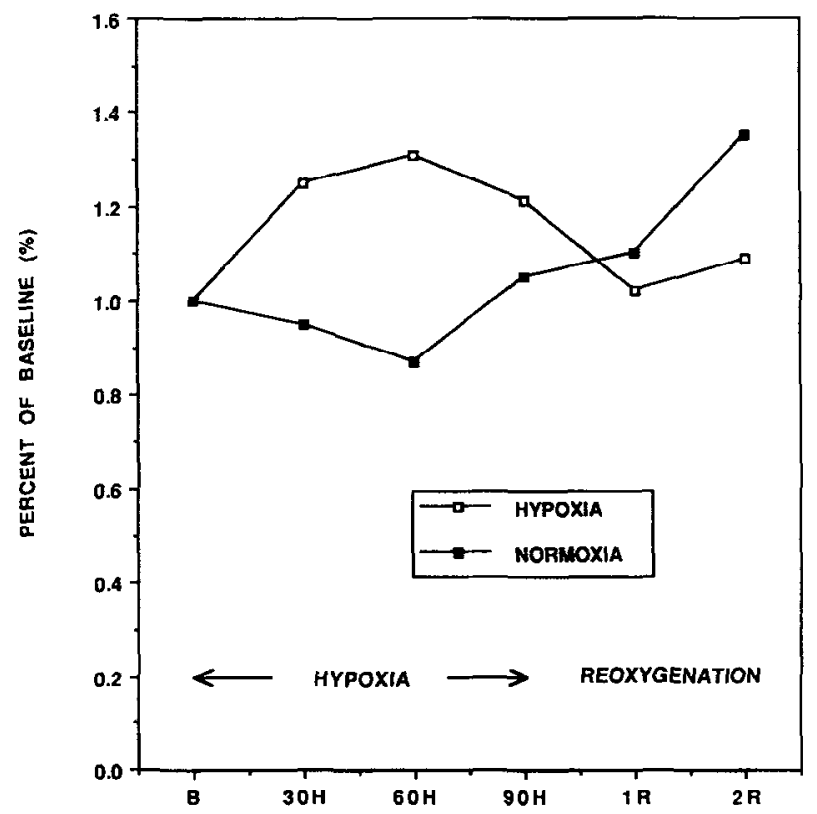

TIME (MINUTES)

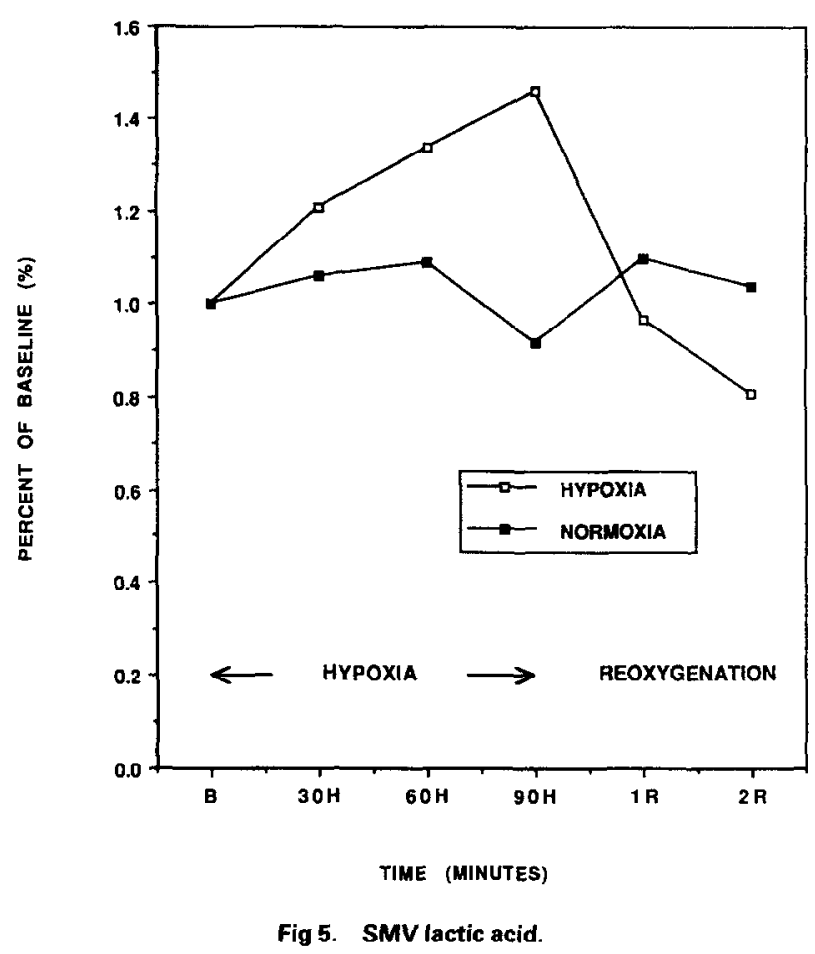

formation or peroxidation of the cellular lipid bilayers.

\section{Mediators}

The arachidonic acid metabolites from the cyclooxygenase pathway, TBX and PGE, and the lipoxygenase pathway, LT were affected differently. TBX was markedly elevated in both groups I and II (Fig 6).

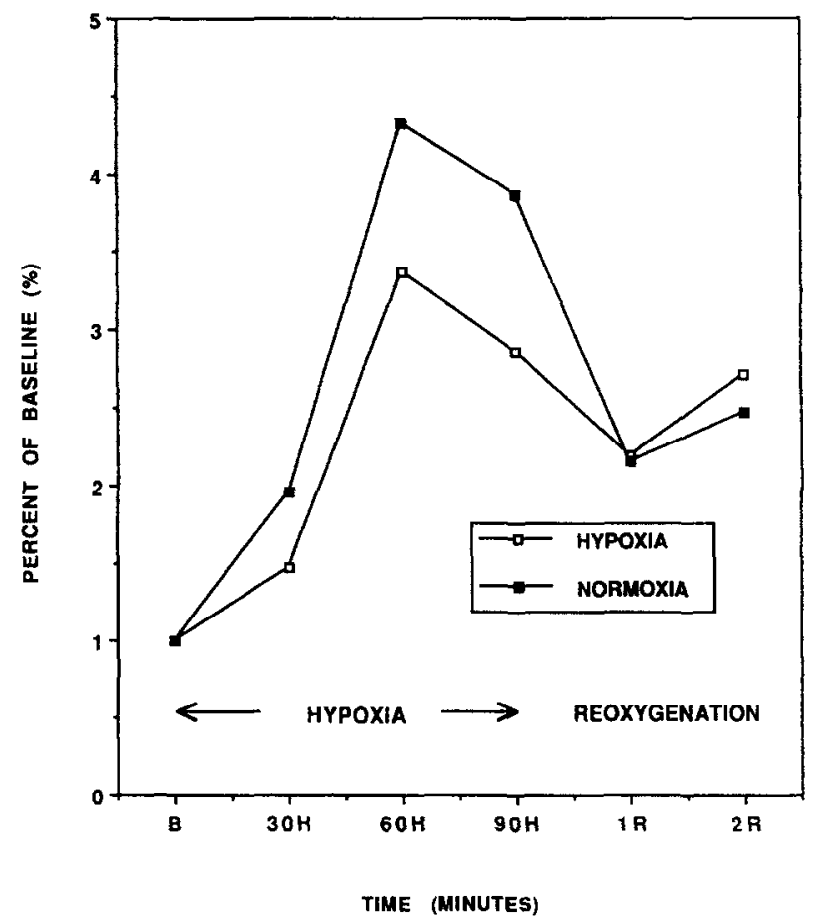

Fig 6. TBX. 
This elevation is most likely related to the stress of the surgical preparation because the levels were similar in both groups and were not affected by hypoxia. The prostaglandins increased late in the reoxygenation phase in group I (Fig 7); however, this was not a statistically significant increase. LTs remained near baseline levels in both groups I and II.

Serum levels of the cytokines TNF and IL-6 were likewise affected differently. TNF levels remained near baseline in both groups I and II (Fig 8). However, IL-6 was markedly elevated in group I compared with group II $(P<.05)$ and remained elevated throughout the experimental study period (Fig 9).

\section{Histological Analysis}

Histological evaluation revealed only minimal changes in intestinal mucosal histology in the group I animals. The intestine of group I animals occassionally demonstrated submucosal edema but to a very minor degree. Mucosal villous architecture was maintained in both group I and group II specimens. Leukocyte infiltration and vascular congestion were not present in either group I or group II at 24 hours after the hypoxia-reoxygenation insult.

\section{Bacterial Translocation}

Group III $(\mathrm{n}=3)$ animals were killed without intervention and bactcrial analysis was performed. No animal in group III had bacteria in the MLNs, spleen, or liver. In group II, bacteria were present in

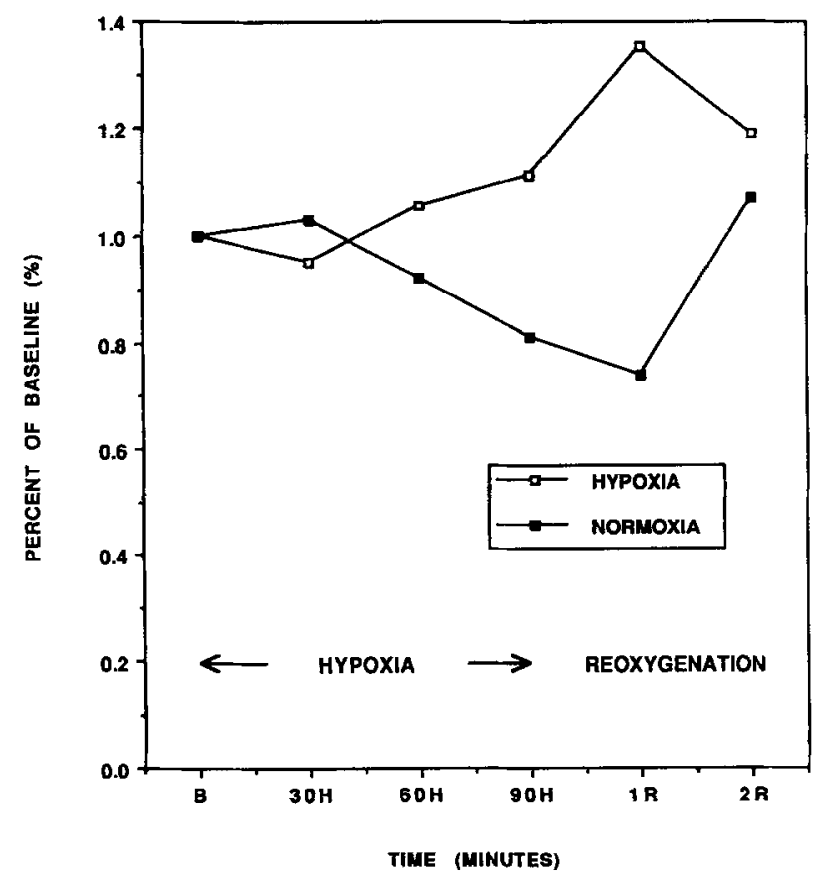

Fig 7. PGE.

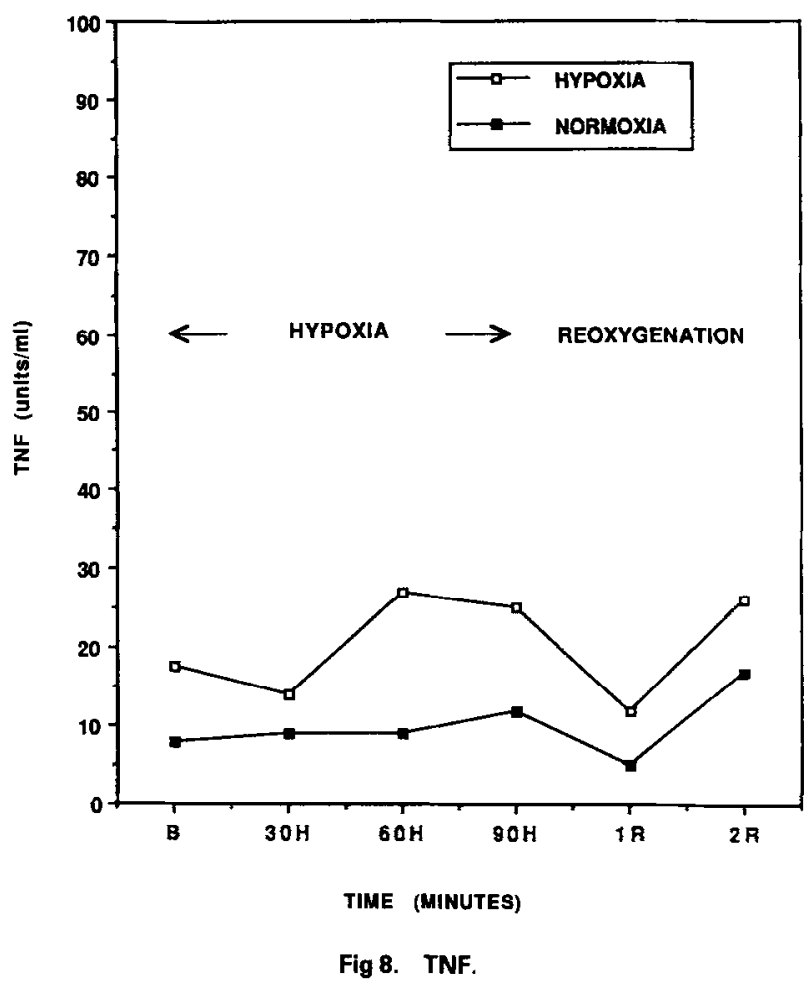

$50 \%$ of the spleen and $33 \%$ of the liver specimens but there were no bacteria in any of the MLN specimens. In contrast, group I animals showed a $67 \%$ incidence of bacteria in the spleen, $33 \%$ incidence in the liver, and a 50\% incidence in the MLNs (Fig 10).

The MLNs of group I cultured $E$ coli, Klebsiella, Streptococcus D, and Salmonella species. The spleen and liver specimens of both groups I and II cultured $E$

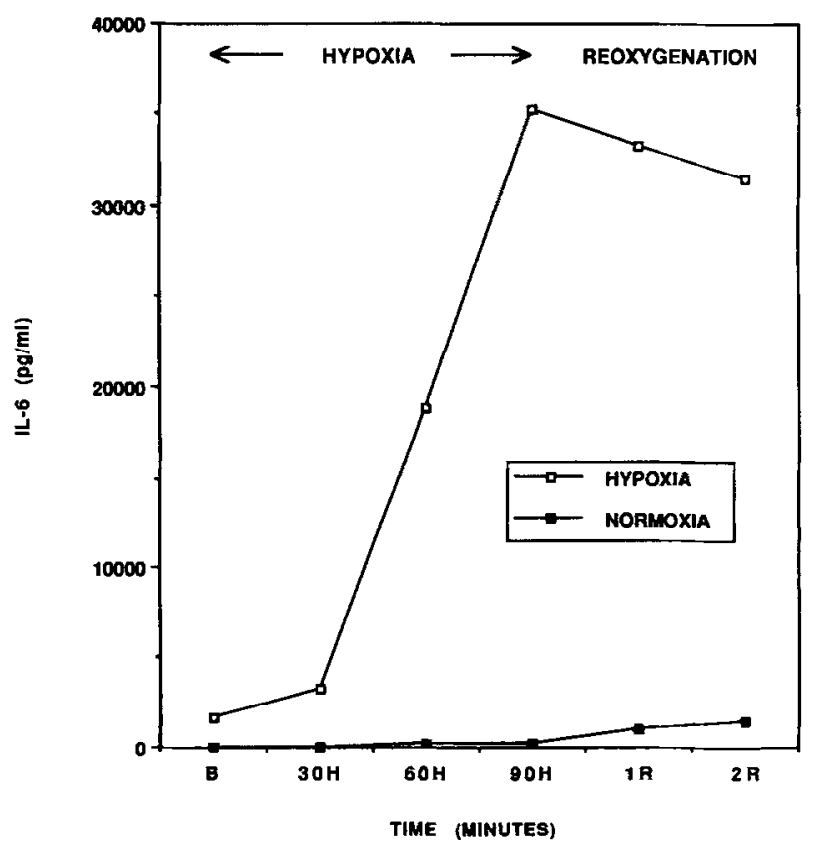

Fig 9. IL-6. 


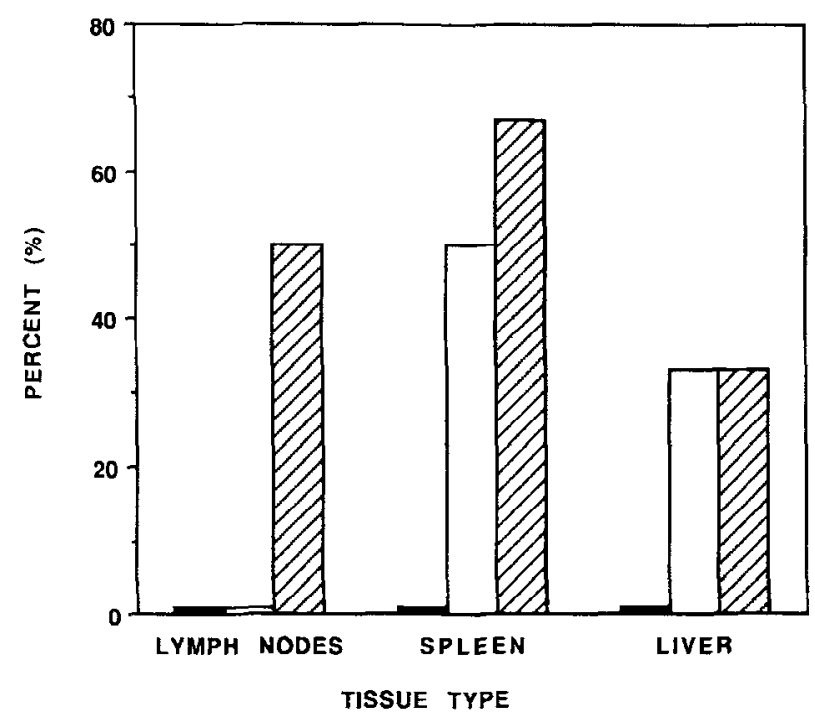

Fig 10. Incidence of bacterial colonization. $\mathrm{B}$, Harvest (group III); $\square$, normoxia (group II); 河, hypoxia (group I].

coli and Klebsiella, but predominantly Staphlococcus species. It appears that a combination of laparotomy, mesenteric cannulation, and manipulation of the bowel led to significant bacterial translocation into the portal system, a process that appeared to be independent of the hypoxic stress. However, the lymphatic system was not affected by bowcl manipulation or laparotomy but was affected by the hypoxiareoxygenation insult. Bacterial density (CFU/g) was similar in the spleen and liver of groups I and II (Fig 11).

\section{DISCUSSION}

Experimental studies of adult feline small bowel exposed to hypoxia have demonstrated the produc-

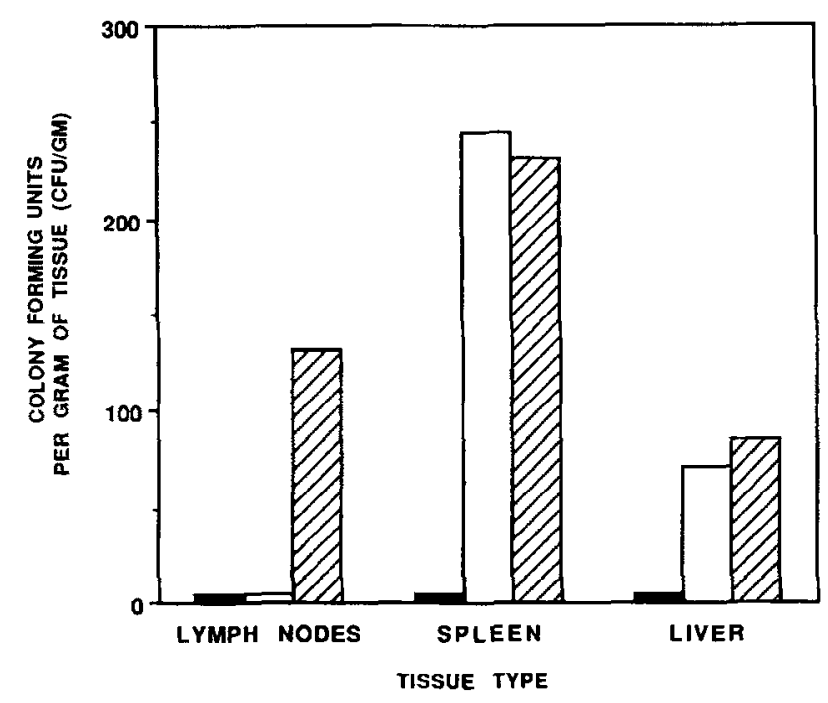

Fig 11. Bacterial density. D. Harvest (group III); $\square$, normoxia (group II); 罚, hypoxia (group I). tion of a mucosal lesion characterized by massive epithelial lifting and denuded villi with disintegration of the lamina propria, hemorrhage, and ulceration. ${ }^{19}$ Hypoxic and hypovolemic injury in puppies has produced microvascular platelet and neutrophil aggregates with endothelial cell disruption and intracellular disorganization of the mucosal cells. ${ }^{20}$ These experimental lesions correlate well with the described histology of human bowel injury. ${ }^{1}$

Experimental tissue damage has been associated with widespread functional impairment and an increased mucosal microvascular permcability to substances with molecular weights between 680 and $68,000 .^{21,22}$ Somc investigators have proposed that it is the immaturity of the neonatal gut that initially allows uptake of macromolecules, thus permitting absorption of toxic products from growing bacteria and finally resulting in intestinal damage. ${ }^{23,24}$

It has been postulated that these injuries represent a spectrum of physiological responses, probably related to the level and duration of hypoxia, the condition of the host, and the timing and effectiveness of resuscitation measures. Superoxide radicals formed during postischemic reperfusion have been directly implicated as one of the elements in the pathogenesis of ischemic mucosal lesions ${ }^{25,26}$ and increased microvascular permeability. ${ }^{6}$

When hypoxia reaches a critical level, depletion of adenosine triphosphate (ATP) stores occurs and anaerobic metabolism ensues. The breakdown of ATP and the release of its high-energy phosphate bond results in the irreversible production of hypoxanthine. When reperfusion oxygen becomes available, hypoxanthine is converted to xanthine and superoxide radicals are generated. Once superoxide radicals are formed and reperfusion oxygen supplied, mucosal injury occurs.

The intestinal mucosa is the major barrier preventing bacteria from gaining access to the gut and systemic organs and tissues. Factors that promote translocation include: disruption of the ecology of the indigenous gastrointestinal microflora leading to bacterial overgrowth, ${ }^{27}$ impaired host immunity, ${ }^{28}$ and physical disruption of the gut mucosal barrier from direct chemical injury, ${ }^{29}$ endotoxin, ${ }^{30}$ and hemorrhagic shock. ${ }^{31}$

Deitch et al have shown, in multiple studies, that the translocation of bacteria to MLNs and, subsequently, to the liver and spleen is dependent on multiple synergistic factors. In health, indigenous bacteria are probably in a state of continuous translocation from the gastrointestinal tract at a very low rate. The translocating bacteria are killed either en 
route through the lamina propria or in situ in the recticuloendothelial organs such as the MLNs. However, with interventions such as oral antibiotics, the ecological equilibrium in the gastrointestinal tract is disrupted and concomitant bacterial translocation occurs but host defenses confine this to the MLNs. With immunosuppression, ${ }^{32}$ hemorrhagic shock, ${ }^{31}$ endotoxin, ${ }^{30}$ or protein malnutrition, ${ }^{33}$ the translocating bacteria get past the MLN to the spleen, liver, and blood stream. These factors enable the bacteria to overpower the host defenses.

Thus, the mucosal injury that occurs in hypoxiareoxygenation states could be expected to enhance bacterial translocation as well. With the natural immunocompromised state of neonates, translocation could occur without significant necrosis of the bowel. It is this intermediate state that we postulated could result in neonatal sepsis, where there is no focal infection or gross organ injury.

To reduce oxygen delivery in our model, we used the most severe systemic hypoxia that could be applied without rapid hemodynamic deterioration occuring. It is the decline in $\mathrm{O}_{2}$ consumption, however, that indicates inadequate oxygen supply at the cellular level, impending anaerobic metabolism and substrate accumulation for free oxygen radical formation. In the present model, mucosal oxygen consumption started to decline only after $\mathrm{O}_{2}$ delivery was reduced by more than $70 \%$. This decline in oxygen consumption did not occur until 60 minutes of hypoxia and was predominately related to a gradually declining mucosal blood flow. Ninety minutes of hypoxia resulted in only 30 minutes of true tissue oxygen deprivation.

The delayed change in mucosal blood flow suggests that metabolic processes at the cellular level are occuring over time and may be the etiology of the decreased mucosal perfusion. With reoxygenation, mucosal blood flow also recovered slowly, again suggesting a time-dependent biochemical process was taking place. The explanation for the overconsumption of oxygen once bowel blood flow did return to baseline is not readily apparent.

Systemic and splanchnic lactic acid levels increased but not to a significant degree, indicating that anaerobic metabolism was not predominant. The absence of a systemic anaerobic state is consistent with the observation that lipid peroxidation byproducts did not rise either. Even though severe systemic hypoxia was induced, it was only in the very late stage that mucosal oxygen consumption declined and anaerobic metabolism began to occur. Free radical-mediated injury was not initiated in this model.

The arachidonic acid metabolites, TBX and PGE, from the cyclooxygenase pathway have well-known hemodynamic effects in models of hypotension and septic shock. In this model, PGE was not significantly elevated between groups I and II. However, TBX was markedly elevated in both groups I and II, suggesting that the laparotomy and bowel manipulation by themselves will initiate TBX production. The hypoxic insult introduced in group I did not alter the TBX clcvation. LTs, from the lipoxygenase pathway, were not altered from baseline in either groups I or II. Thus, arachidonic acid mctabolites do not appear to be significantly induced during hypoxia.

TNF was not elevated in either group I or group II to a significant degree. However, IL-6 levels were markedly elevated in group I during hypoxia. Prcvious investigations have concluded that the formation of IL-6 is the result of elevation in TNF and that these cytokines cause hemodynamic instability. ${ }^{34}$ This model demonstrated a hypoxia-driven elevation of IL- 6 independent of TNF or any of the arachidonic acid metabolites. However, the elevation in IL-6 did not result in any changes in hemodynamic parameters.

Only minimal histological injury could be demonstrated in group I animals. This minimal injury is to be expected because anaerobic metabolism and lipid peroxidation were not initiated until very late. The minimal submucosal edema that was demonstrated in some of the specimens is the earliest sign of mucosal injury.

Even though anaerobic metabolism did not occur and significant histological disruption of the mucosa could not be generally demonstrated, bacterial translocation of aerobic species to MLNs resulted from hypoxia. Bacteria were found in the liver and spleen of both groups I and II. It appears that manipulation and cannulation of the bowel portal circulation may explain the high bacterial load in the normoxia and hypoxia groups. The data from these experiments support the conclusion that hypoxia is capable of allowing bacterial translocation through intact mucosal barriers and into the lymphatic system. Additionally, this study has shown that, even under severe and prolonged hypoxic conditions, the small bowel mucosa is capable of maintaining aerobic metabolism and avoiding a reperfusion/reoxygenation injury. The addition of an ischemic injury to hypoxia in this model would certainly have initiated anaerobic metabolism earlier and a significant reoxygenation injury would most likely have occurred. 


\section{REFERENCES}

1. Wayne ER, Burrington JD, Hulter J: Neonatal necrotizing enterocolitis. Arch Surg 110:476-480, 1975

2. Touloukian RJ, Berdon WG, Amoury RA, et al: Surgical experience with neonatal necrotizing enterocolitis in the infant. $J$ Pediatr Surg 2:389-401, 1967

3. Touloukian RJ, Posch JN, Spencer R: The pathogenesis of ischemic gastroenteritis of the neonate: Selective gut mucosal ischemia in asphyxiated neonatal piglets. J Pediatr Surg 7:36-43, 1973

4. Kosloske AM. Ulrich JA: A bacteriologic basis for the clinical presentation of necrotizing enterocolitis. J Pediatr Surg 15:558564,1980

5. Barlow B, Santulli TV: An experimental study of necrotizing enterocolitis-The importance of breast milk. J Pediatr Surg 9:587-595, 1974

6. Book LS, Herbst JJ, Jung AL: Necrotizing enterocolitis in low-birth-weight infants fed an elemental formula. J Pediatr 87:602-605, 1975

7. Caralos-Riera JM, Coch BD: Bowel perforation after exchange transfusion in the nennate: Review of the literature and report of a case. Surgery $68: 895-897,1970$

8. Hardy JD, Sauage TR, Shizodaria C: Intestinal perforation following exchange transfusion. Am J Dis Child 124:136-141, 1972

9. Touloukian RJ, Kadar A, Spencer RP: The gastrointestinal complications of neonatal umbilical venous exchange transfusion: A clinical and experimental study. Pediatrics 51:36-43, 1973

10. Nagaraj HS, Sander AS, Cook LN, et al: Gastrointestinal perforation in very low birth weight infants following indomethicin therapy. J Pediatr Surg 16:1003-1007, 1981

11. Barker SB, Summerson WH: Colorimetric determination of lactic acid in biological material. J Biol Chem 138:535-554, 1941

12. Beuge JA, Aust SD: Microsomal lipid peroxidation, in Methods of Enzymology: Biological Oxidations, Microsomal, Cytochrome P-450 and Other Hemoprotein Systems, vol 52. New York, NY, Academic, 1978

13. Espevik T, Nissen-Meyer J: A highly sensitive cell line, WEHI 164. clone 13, for measuring cytotoxic factor/tumor necrosis factor from human monocytes. J Immunol Meth 95:99-105, 1986

14. Eskandari MK, Nguyen DT, Kunkel SL, et al: WEHI 164 subclone 13 assay for TNF: Sensitivity, specificity and reliability. Immunol Invest 19:69-79, 1990

15. Aarde LA, DeGrott ER, Schaap IL, et al: Production of hybridoma growth factor by human monocytes. Eur J Immunol 17:1411-1416, 1987

16. Hitzpatrick FA, Bundy GL: Hapten mimic elicits antibodies recognizing prostaglandin $\mathrm{E}_{2}$. Proc Ntl Acad Sci 75:2689-2693, 1978

17. Fitzpatrick FA, Gorman RR, McGuire JC, et al: A radioimmunassay for thromboxane $B_{2}$. Anal Biochem 82:1-7, 1977
18. Ford-Hutchinson AW, Bray MA, Doig MV, et al: Leukotriene $B$, a potent chemokinetic and aggregating substance released from polymorphonuclear leukocytes. Nature 286:264-265, 1980

19. Granger DN, Rutili G, McCord JM: Superoxide radicals in feline intestinal ischemia. Gastroenterology 81:22-29, 1981

20. Harrison MW, Connell RS, Cambell JR, et al: Fine structural changes in the gastrointestinal tract of the hypoxic puppy: A study of the natural history. J Pediatr Surg 12:403-407, 1977

21. Grogaard B, Parks DA, Granger DN, et al: Effects of ischemia and superoxide radicals on mucosal albumin clearance in the dog intestine. Am J Physiol 5:448-454, 1982

22. Kingman JG, Whorwell RJ, Loehry CA: Small intestinal permeability: Effects of ischemia and exposure to acetyl salicylate. Gut 17:354-361, 1976

23. Lawrence G, Bares J, Gaul A: Pathogenesis of neonatal necrotizing enterocolitis. Lancet 1:137-139, 1982

24. Gray ES, Lloyd DJ, Hurne CH: Pathogenesis of neonatal necrotizing enterocolitis. Lancet 1:451, 1982

25. Parks DA, Bulkley GB, Granger DN, et al: Ischemic injury in the cat small intestine: Role of superoxide radicals. Gastroenterology 82:9-15, 1982

26. Schoenberg $M$, Younes $M$, Muhl E, et al: Free radical involvement in ischemic damage of the small intestine, in Greenwald $R$, Cohen $G$ (eds): Proceedings of the 3 rd International Conference on Superoxides and Superoxide Dismutase. New York, NY, Elsevier/North Holland, 1983, pp 154-157

27. Berg RD: Inhibition of Escherichia coli translocation from the gastrointestinal tract by normal cecal flora in gnotobiotic or antibiotic-decontaminated mice. Infect Immun 29:1073-1081, 1980

28. Berg RD: Bacterial translocation from the gastrointestinal tracts of mice receiving immunosuppressive chemotherapeutic agents. Curr Microbiol 8:285-292, 1983

29. Morehouse J, Specian R, Stewart J, et al: Promotion of the translocation of indigenous bacteria of mice from the GI tract by oral ricinoleic acid. Gastroenterology 91:673-682, 1986

30. Deitch EA, Berg R, Specian R: Endotoxin promotes the translocation of bacteria from the gut. Arch Surg 122:185-190, 1987

31. Baker JW, Deitch EA, Berg RD, et al: Hemorrhagic shock induces bacterial translocation from the gut. J Trauma 28:896-906, 1988

32. Berg RD, Wommack E, Deitch EA: Immunosuppression and intestinal hacterial nvergrowth synergistically promote bacterial translocation. Arch Surg 123:1359-1364, 1988

33. Deitch EA. Winterton J, Berg R: Effect of starvation, malnutrition and trauma on the gastrointestinal tract flora and bacterial translocation. Arch Surg 122:1019-1024, 1987

34. Waage A, Rrandtzaeg $P$, Halstensen A, et al: The complex pattern of cytokines in serum from patients with meningococcal septic shock. J Exp Med 169:333-338, 1989

\section{Discussion}

Robert Arensman (Chicago, IL): It is very interesting to me that this study shows that the experimental animals, subjected to what most of us would consider a rather severe hypoxic stress, were showing the process of bacterial translocation, and yet there were no major metabolic derangements in these puppies. The investigators were not able to detect major mucosal damage in these particular animals, and yet there was a major change in the amount of bacterial translocation. 
In the past, we looked on the appearance of portal air as a very poor prognostic sign in children with necrotizing enterocolitis. We were surprised to discover with Doppler that air in the portal system was actually an early and common sign of necrotizing enterocolitis. These last two papers are showing once again that our ability to detect and measure subtle alterations in the delivery and consumption of oxygen may be the very early signs of hypoxia/reperfusion problems.

Certainly the conclusion here is that there were major derangements in these puppies with very minimal microscopic findings. How do you see that we can apply this information we are hearing this morning to more carefully identify and monitor children who are at great risk for hypoxic episodes, particularly children likely to develop necrotizing enterocolitis or children likely to suffer hypoxia associated with surgical stress or severe trauma?

Robert Foglia (St Louis, MO): You describe a decrease in your blood flow with return to oxygenation. Would you not expect to see increased blood flow? Could capillary endothelial swelling due to edema be the factor which has limited your blood flow during your reoxygenation period? Would that delay the change in blood flow?

Burton Harris (Boston, MA): This is really a powerhouse paper, and I congratulate the authors for their experimental design and for the way in which the experiment was carried out. They touched every conceivable base.

Two questions: first, did you culture the stool to be sure that the organisms you were finding came from the bowel; and second, do you have any plans to go back and use this model with blocking agents to some of the potential mediator compounds?

David Collins (San Diego, CA): I think I recall from medical school physiology that the dog normally has bacteria in its liver so I am not sure how much it contributes to the problem to assess that factor. I would also like to ask the authors if the puppies had any signs of discomfort while being severely deprived of oxygen and was there any form of sedation or anesthesia used?

Donald Nuss (Norfolk, VA): Did you do any blood cultures on any of the specimens?

J. Lelle (response): Previous models of bowel injury have used arterial clamping or occlusion; however, there is no evidence that neonates undergo this type of insult prior to the development of necrotizing enterocolitis or neonatal sepsis. There is, however, evidence that hypothermia and hypoxia occur commonly and to varying degrees. This study was conducted to look at one isolated stress and hypoxia was the one chosen.

There is some question in the literature as to how well fetal bowel mucosa responds to physiological stresses when compared with infant, adult, or premature mucosa. This experiment used an infant model and represents a pilot study. Further studies compare the physiological responses of other age groups are needed.

Blood cultures were done on all animals; however, no positive cultures were demonstrated in any animal. Stool was cultured in most but not all animals. We found that the bacterial levels were consistent with those reported by Deitch and others to be sufficient to allow bacterial translocation under some types of stresses but not spontaneously.

The bacteria noted in the MLNs were enterics, predominantly $E$ coli and Staphylococcus species. There was no anaerobic bacterial translocation in any animals. The bacteria found in the spleen and liver specimens were mostly $S$ epidermidis and were felt to be contamination from our system of cannulating the mesenteric vessels and manipulation within the abdomen.

The animals were anesthetized completely during the entire study period with intermittent doses of pentobarbital and were without discomfort. No animal demonstrated diarrhea or any form of sepsis preoperatively or postoperatively in the 24 -hour observation period. These animals were "acute" and not conditioned animals. However, any animal that presented with diarrhea prior to the experiment was eliminated from the study.

In response to the theory that interstitial swelling caused the delayed change in mucosal blood flow, it should be noted that mucosal blood flow was found to be back to normal in all animals after 2 hours. Therefore, one would have to hypothesize that interstitial fluid swelling could occur and return back to baseline within 2 hours. While that may be the case, we have no evidence in this model to prove or disprove this theory, although I feel that this is an unlikely explanation. 